\title{
Estudiantes afrocolombianos en la Universidad de Nariño: prejuicios, estereotipos y situaciones de discriminación ${ }^{1}$
}

\author{
María Fernanda Martínez Hoyos ${ }^{2}$ \\ Mauricio Pantoja Obando ${ }^{3}$, Ana Lucia Caicedo Leitón ${ }^{4}$
}

\begin{abstract}
Resumen
Este artículo surge de la investigación realizada por el grupo de investigación Psicología y Salud de la Universidad de Nariño- Colombia, cuyo objetivo fue comprender los prejuicios y estereotipos que se presentan entre estudiantes pertenecientes (EP) y no pertenecientes (ENP) a comunidades afrocolombianas, indígenas y homosexuales y sus implicaciones en este contexto universitario. Se encontró que la comunidad homosexual presenta los mayores prejuicios, estereotipos y manifestaciones de discriminación, seguida de la comunidad Afrocolombiana, siendo esta última a la cual se enfoca el presente artículo.

Se desarrolló un proceso de investigación cualitativa mediante la conformación de 12 grupos focales: Cuatro conformados por estudiantes universitarios que se reconocieron como pertenecientes a comunidades afrocolombianas y ocho con estudiantes no pertenecientes a ésta. La información se analizó mediante el programa Atlas-Ti desde la perspectiva psicosocial de las actitudes, identificando prejuicios y estereotipos positivos y negativos que manifiestan los estudiantes universitarios hacia sus compañeros afrocolombianos y las manifestaciones de discriminación sutil o manifiesta que de estos se derivan, además se analizan causas individuales y socioculturales atribuidas por los participantes a las situaciones de discriminación y se plantean alternativas para el abordaje de la problemática en el contexto universitario.
\end{abstract}

Palabras clave: Contexto universitario, diversidad, prejuicios, estereotipos, discriminación, estudiantes afrocolombianos, racismo, integración.

1 Recibido: 11 de diciembre de 2014. Aceptado: 22 de febrero de 2015.

2 María Fernanda Martínez Hoyos, Psicóloga de la Universidad de Nariño y Magister en Educación desde la Diversidad de la Universidad de Manizales, docente de la Maestría en Educación desde la Diversidad de la Universidad de Manizales, Orientadora Escolar de la Institución Educativa Municipal Heraldo Romero Sánchez de la ciudad de Pasto y miembro del grupo de investigación Psicología y Salud de la Universidad de Nariño, Correo electrónico: mariafermatinez13@gmail.com.

3 Diego Mauricio Pantoja Obando, Psicólogo Universidad de Nariño, Técnico en Criminalística e Investigador Forense Judicial Escuela Colombiana de Criminalística, miembro del grupo de investigación Psicología y Salud de la Universidad de Nariño, Psicólogo Fundación ATME, Correo electrónico: diego7mau@yahoo.es.

4 Ana Lucia Caicedo Leitón, Psicóloga de la Universidad de Nariño, miembro del grupo de investigación Psicología y Salud de la Universidad de Nariño, ParqueSoft pasto - líder de empoderamiento de talento humano. correo electrónico anitakley@hotmail.com 


\section{Abstract}

\section{Afro-Colombian Classmates in university context: prejudice, stereotyping and discrimination situations.}

This article comes from research made at Nariño University - Colombia by the Psychology and Health Investigation group. It was focused on prejudice and stereotyping that may exist between students belonging (EP) and not belonging (ENP) to Afro, Indigenous and Homosexual community, and its implications in the university context. Was discovered that Homosexual community experiences major prejudices, followed by the Afro-Colombian. This document is focus on Afro- Colombian results.

The study was developed from the qualitative paradigm; information was collected through 12 focus groups, four of them conformed by Afro-Colombian self-recognized students, and eight groups conformed by other population. Information was analyzed using Atlas-Ti software from the psico-social perspective of attitudes, identifying positive and negative prejudices and stereotypes that students manifest to their Afro- Colombian classmates, and the subtle or overt ways of discrimination derived from this. Also analyzed the individual and sociocultural causes attributed to discrimination situations, and propose alternatives to approach this problem in university context.

Keywords: Univertisty context, diversity, prejudice, stereotypes, discrimination, Afro-Colombian students, racism, integration.

\section{Presentación}

La investigación de la que se deriva el presente artículo parte de la necesidad planteada en el grupo de investigación Psicología y Salud de la Universidad de Nariño de iniciar procesos investigativos hacia dentro de la propia Universidad y no solo hacia fuera de esta como usualmente se realizan, los resultados que se presentan se derivan de una investigación desarrollada entre los años 2010 y 2012 en la que se buscó responder a la pregunta ¿Cómo son los prejuicios y estereotipos que se presentan hacia estudiantes de la Universidad de Nariño pertenecientes (EP) y no pertenecientes (ENP) a comunidades afrocolombianas, indígenas y homosexuales y cuáles son sus implicaciones en el contexto universitario?

\section{Develando el problema}

El análisis de antecedentes a nivel internacional, nacional y regional deja en evidencia las múltiples y variadas manifestaciones de discriminación de las que suelen ser receptoras las comunidades afrodescendientes, es importante reconocer que estas manifestaciones se sustentan en prejuicios y estereotipos que se han construido socialmente y se han consolidado históricamente, arraigándose en el imaginario colectivo lo que las hace difíciles de trasformar; cómo no hacer referencia por ejemplo al fenómeno de la esclavitud o el apartheid como máximas expresiones históricas de discriminación, prácticas sustentada en fuertes creencias erróneas promovidas para mantener el poder y la dominación durante la colonia y que han sido construidas en torno a la categoría "negro", para Wabgou (2009) el rechazo contemporáneo a esta categoría se debe a que fue establecida como un instrumento de explotación creada por los blancos europeos, para garantizar una superioridad supuestamente natural que justificaba el sometimiento de todos aquellos 
que no compartieran sus características, bajo esta mirada del Otro "lo negro era (y lo sigue siendo) visto como lo inferior, lo bárbaro, lo malo y que en el mejor de los casos, había que civilizar" (Wabgou, 2009, p.54) creencias que tienen gran impacto en el presente de las comunidades afrodescendientes.

En Colombia, la Constitución Política de 1991 (Artículo 13) reconoce la diversidad étnica y cultural al afirmar que todas las personas gozarán de los mismos derechos, oportunidades y libertades, sin ninguna discriminación por razones de sexo, raza, origen nacional o familiar, lengua, religión, opinión política o filosófica, sin embargo, para Friedemann (1993, citada por Wabgou, 2009), este reconocimiento se da en medio de una serie de limitaciones teóricas ya que en los años precedentes el interés antropológico había estado concentrado en las comunidades indígenas, marcando el desconocimiento del carácter etnicista de "lo negro" y evidenciando según la autora, que la Antropología - y en general la academia- desarrollada antes de los años ochenta, "había sido cómplice de un olvido consciente de la cultura y la sociedad negra, como parte importante de la sociedad colombiana"(p. 29), por lo tanto el reconocimiento de la diversidad étnica y cultural del país se da en medio de la "ausencia de una "identidad institucionalizada del negro" que en contraste sí existía para las comunidades indígenas desde la Colonia" (Friedemann citada por Wabgou, 2009, p. $30)$. Este vacío en relación a la identidad y cultura afrocolombiana genero a partir de 1991 múltiples debates de los que se han derivando una serie de leyes y decretos entre los que cabe resaltar la Ley 70 de 1993, que hace referencia a la ubicación y conformación de territorios comunales para las comunidades negras y plantea el derecho a un proceso educativo acorde con sus necesidades y aspiraciones etnoculturales; el Decreto 1122 de 1998, que expide normas para el desarrollo de la Cátedra de Estudios Afrocolombianos en todos los establecimientos de educación formal del país y la Ley 1482 de 2011 que busca garantizar la protección de los derechos de toda persona que puedan ser vulnerados a través de actos de racismo o discriminación.

El reconocimiento de este panorama histórico, jurídico y político caracterizado por la invisibilización, discriminación y exclusión de las comunidades afrocolombianas desde la colonia hasta nuestros días, valida la pregunta por la forma actual que adquieren los prejuicios y estereotipos hacia esta comunidad, concretamente en un contexto académico como el universitario, al reconocer su contribución al silenciamiento y el olvido de estas poblaciones, al respecto, Mosquera (1987, citado por Castillo y Caicedo, 2014) plantea que

... la educación es reproductora de prejuicios raciales, es reproductora de una sicología social que inferioriza y subvalora a las comunidades negras al no reconocerlas como sujetos protagonistas de la historia y de la construcción nacional de estas naciones, sino solamente objetivizarlas como esclavas; ahí empieza y termina la historia de nuestras comunidades... (p.39).

Por su parte Meneses (2013) al hacer referencia a las representaciones sociales que actualmente tiene el profesorado sobre etnoeducación y la cátedra de estudios afrocolombianos considera que se debe

superar el matoneo epistémico afrodescendiente y la racialización del saber, que mediante cuerpos ideológicos perturban la afrodescendencia en la escuela y la universidad, por tanto imposibilitan o parcializan la pluridiversidad bajo una capa del indigenismo histórico de la academia colombiana (p.62)

Por lo tanto, a pesar de que en el escenario jurídico se ha incrementado la preocupación por la discriminación, especialmente por la imperante lucha y procesos de resistencia de los grupos 
susceptibles de ser discriminados, se reconoce que su discusión en el ámbito académico es muy débil y "todavía, existen pocos estudios en Colombia que examinen teóricamente y documenten empíricamente el fenómeno de la discriminación" (Viveros, 2007, p. 108).

\section{Explorando el camino recorrido}

A pesar de los avances que se han presentado en el campo legislativo, son múltiples los estudios y reportes de organizaciones internacionales (ONU, 2012) y nacionales (Observatorio contra la discriminación y el racismo, 2012), que demuestran la frecuencia con que poblaciones culturalmente diversas en el contexto regional, nacional e internacional son víctimas de estigmatización y de formas de exclusión social como el racismo y la xenofobia, además de experimentar dificultades para acceder a la satisfacción de sus derechos fundamentales como salud, educación, trabajo, el respeto por sus prácticas y tradiciones culturales, la propiedad colectiva y el patrimonio. En el contexto latinoamericano, de acuerdo con París (2002), diversos estudios han demostrado que las manifestaciones de discriminación y rechazo hacia ciertas poblaciones "minoritarias" ya sea por sus características étnicas, sexuales o culturales guardan una estrecha relación con la presencia de prejuicios y estereotipos de carácter negativo, fundamentados principalmente en la amenaza que representan para el mantenimiento de las normas, valores y "buenas costumbres" que sustentan la estructura social de la cultura dominante y por ende para el mantenimiento de su poder.

En relación al contexto nacional el Observatorio de Discriminación Racial en su informe del año 2009 indica que el $10,6 \%$ de la población se auto-reconoce como afro descendiente, siendo ésta población una de las principales víctimas de la discriminación racial y del racismo que se expresa en violaciones constantes a sus derechos, profundizando el ciclo de marginalidad, pobreza e invisibilidad en el que se encuentran sumidos, sumado al hecho de ser una población particularmente afectada por el conflicto armado, la violencia y el desplazamiento forzado, lo cual revela la existencia y persistencia de un racismo estructural del estado.

También se destaca el análisis de diferentes estudios sociales sobre la población afrocolombiana, realizado por Cavelier (2007) quien encontró que las discusiones de la academia en torno al tema de la discriminación, exclusión y situaciones de marginalidad que las personas negras experimentan diariamente en Colombia son relativamente recientes y la mayoría pueden encontrarse en la última década en la que se presentó una tendencia a tratar el tema. Este vacío en el conocimiento, se hace más evidente en relación a las manifestaciones de discriminación en contextos académicos como el universitario, aunque, se destacan estudios realizados en otros contextos que contribuyen a la comprensión de la problemática como el de Wabgou (2009) que aborda el tema de las migraciones de las comunidades afrocolombianas a la ciudad de Bogotá, mostrando como la discriminación y la exclusión social, están marcando la vida cotidiana de la población negra en la localidad de Kennedy; el estudio realizado por Viáfara y Urrea (2006) en las ciudades de Cali, Cartagena y Bogotá que muestra los efectos de la raza y el género en el logro educativo y el estatus socio-ocupacional al indicar que:

La estructura de oportunidades para los individuos negros-mulatos, en especial para las mujeres, no sólo se explica por sus orígenes sociales y niveles educativos inferiores, sino también por un proceso de obstáculos o desventajas acumulativas que pueden ser interpretados como discriminación (Viáfara y Urrea, 2006, p.155)

Se encuentran además los estudios desarrollados por Restrepo (2003, 2004) 
sobre las comunidades negras en Colombia en los que se argumenta que problemáticas como la aquí planteada tienen alcances estructurales que atraviesan nuestras acciones y pensamientos cotidianos de formas que pueden pasar desapercibidas para nosotros mismos, lo que conlleva a que sean difíciles de visualizar tanto para quienes los ejercen como para quienes son sus receptores. Al respeto Viveros (2007) plantea que

En un contexto social como el colombiano, en el que se niega cotidianamente la existencia del racismo, es difícil pensar sobre la discriminación étnico-racial. En efecto, en nuestro país las manifestaciones de racismo casi nunca son espectaculares y la discriminación racial se practica a nivel individual, bajo una forma más de exclusión que de agresión. Por esta razón son escasas las reflexiones sobre la discriminación, la intolerancia y el racismo, tal como son vistos y experimentados por la sociedad colombiana. (Viveros, 2007, p.109).

En el caso concreto de la Universidad de Nariño que para el periodo A del año 2011, contaba con 685 estudiantes afrocolombianos, correspondientes al $7 \%$ de la población estudiantil, se encontró como antecedente investigativo el estudio realizado desde el programa de sociología por Hernández y Meza (2008), quienes afirman que la población afrodescendiente es receptora de prejuicios y manifestaciones de discriminación dentro de ésta Universidad, y señalan la ausencia de estudios sobre esta problemática en las bases de datos regionales vacío en el conocimiento que perdura hasta la fecha y que orientó el carácter exploratorio de la presente investigación.

Los estudios mencionados indican que la problemática planteada se presenta en todos los ámbitos de la realidad nacional e internacional, desde los aspectos jurídicos y normativos hasta las interacciones cotidianas de los contextos laborales, las familias y las instituciones educativas, estas últimas encargadas de gran parte del proceso de socialización de los sujetos, lo que en conjunto brinda argumentos teóricos y prácticos que justificaron el desarrollo de la presente investigación.

\section{Reconociendo los conceptos centrales}

Para comprender la problemática planteada es importante reconocer los conceptos de prejuicio, estereotipo y discriminación que se analizarán brevemente.

De manera general los estereotipos "representan una forma de simplificar la extraordinaria complejidad del mundo social en el que habitamos, al colocar a las personas dentro de categorías" (Gross, 2004, p. 490); de acuerdo con Gómez (2007, p.214) parece existir consenso en que son "esquemas cognitivos, creencias compartidas sobre un grupo que sirven como ahorro de energía para explicar la realidad social".

El origen de los estereotipos se puede ubicar en una compleja interacción que involucra aspectos individuales, interpersonales y sociales, al respecto Molero (2007, p. 603) realiza una revisión de diversas teorías entre las cuales se destaca el enfoque cognitivo que plantea el procesamiento de la información, los procesos de categorización, las correlaciones ilusorias y la ilusión de homogeneidad del exogrupo como elementos de análisis para comprender este fenómeno. El primero hace referencia a la tendencia de la mente humana a analizar la información y tomar decisiones agrupando los estímulos que provienen del exterior en categorías, el segundo se relaciona con la tendencia a sobrevalorar el grado de asociación entre dos variables o acontecimientos sobreestimando por ejemplo la proporción de comportamientos negativos de personas que pertenecen a grupos minoritarios y el tercero con la tendencia a percibir a los miembros de otros grupos como más parecidos de lo que son en realidad, lo cual conduce a considerar 
que todos los integrantes de un grupo determinado son iguales.

El prejuicio por su parte fue definido en 1954 por Allport (citado en Molero, 2007, p.592) como "una actitud hostil o desconfiada hacia una persona que pertenece a un grupo, simplemente debido a su pertenencia a dicho grupo", desde esta perspectiva se concibe el prejuicio como el rechazo de una persona por su pertenencia a un grupo determinado, por su parte, Oskamp (1991) argumenta que el prejuicio es una actitud desfavorable, intolerante, injusta e irracional hacia un grupo social que se puede expresar como un sentimiento desfavorable respecto a un individuo o un grupo y que generalmente es muy fuerte y difícil de cambiar. Al respecto, es importante destacar la teoría planteada por Meertens y Pettigrew (citados en Morelo, 2007b) quienes establecen la diferencia entre el prejuicio manifiesto y el prejuicio sutil, para estos autores el prejuicio manifiesto es cercano y directo, se basa en una percepción de amenaza por parte del exogrupo de la que se deriva un rechazo hacia él y una negación a establecer contacto íntimo con sus miembros, por su parte, el prejuicio sutil es distante e indirecto, se relaciona con la defensa de los valores tradicionales, la exageración de las diferencias culturales y la negación de emociones positivas.

Finalmente la discriminación, de acuerdo con Rodríguez (2005, p.19) puede ser definida como

\section{...una conducta culturalmente}

fundada, sistemática y socialmente

extendida de desprecio contra una persona o grupo de personas, sobre la base de un prejuicio negativo o un estigma asociado con una desventaja inmerecida y que tiene por efecto (intencional o no) dañar sus derechos y libertades fundamentales.

Para García (2002), la discriminación sería el tratamiento diferencial de las personas de acuerdo con su clasificación como miembros de categorías particulares tales como; raza, sexo, edad, clase social, etc. y constituye el proceso por el cual uno o varios miembros de un grupo social determinado es o son tratados de manera diferente, generalmente de manera injusta por pertenecer a ese grupo; de manera específica la discriminación racial según lo contenido en el Artículo 1 de la Convención Internacional sobre la Eliminación de todas las Formas de Discriminación Racial (1965, p.2) se define como

...toda distinción, exclusión, restricción o preferencia basada en motivos de raza, color, linaje $u$ origen nacional o étnico que tenga por objeto o por resultado anular o menoscabar el reconocimiento, goce o ejercicio, en condiciones de igualdad, de los derechos humanos y libertades fundamentales en las esferas política, económica, social, cultural o en cualquier otra esfera de la vida pública.

El reconocimiento de estos conceptos junto al planteamiento de la problemática descrita dio origen a la siguiente pregunta y objetivos de investigación.

\section{Problema de investigación}

¿Cómo son los prejuicios, estereotipos y manifestaciones de discriminación que se presentan en la universidad de Nariño hacia estudiantes pertenecientes a comunidades afrocolombianas?

\section{Objetivos}

\section{Objetivo general}

Analizar los prejuicios, estereotipos y manifestaciones de discriminación que se presentan entre estudiantes de la Universidad de Nariño pertenecientes y no pertenecientes a comunidades afrocolombianas en el contexto universitario.

\section{Objetivos específicos}

Describir las características particulares que adquieren los prejuicios, estereotipos 
y manifestaciones de discriminación hacia la población afrocolombiana en el contexto universitario.

Reconocer los elementos psicosociales que se relacionan con el origen y mantenimiento de prejuicios, estereotipos y manifestaciones de discriminación en el contexto universitario.

Identificar alternativas frente a los prejuicios, estereotipos y manifestaciones de discriminación en el contexto universitario.

\section{Ruta metodológica y participantes}

El estudio se desarrolló desde el paradigma cualitativo por considerarlo el más apropiado para abordar la problemática planteada que enmarca aspectos sociales, culturales, subjetivos e intersubjetivos. Reyes (1999, p. 13) argumenta que éste paradigma de investigación pretende "entender y describir una escena social y cultural desde adentro, desde la perspectiva émica", es decir, desde la perspectiva de los individuos que configuran esa escena social, mediante la interpretación de los procesos sociales que se derivan de la interacción. Por esta razón, se desarrolló la técnica de grupos focales que de acuerdo con Prieto y March (2002) pretende conseguir información en profundidad sobre lo que las personas opinan y hacen, explorando los por qué y los cómos de sus opiniones y acciones, y trabajando desde los discursos, conversaciones e interacciones de los grupos. Para el desarrollo de los grupos se diseñó el siguiente protocolo en el que se especifica el procedimiento a seguir:
Se desarrollaron en total 12 grupos focales, 4 conformados por estudiantes pertenecientes a la organización estudiantil Somos Pazcífico de la Universidad de Nariño (EP) integrada por estudiantes que se reconocen a sí mismos como afrocolombianos y 8 por estudiantes no pertenecientes a comunidades afrocolombianas de esta Institución (ENP) si bien la categoría de genero no fue un criterio de exclusión para la participación en estos grupos todos los grupos estuvieron conformados por hombres y mujeres, participando en total 32 hombres y 54 mujeres en todo el estudio de los cuales 19 hombres y 13 mujeres se reconocen como afrocolombianos.

\section{Discusión}

La figura 2 sintetiza la relación entre las categorías deductivas y emergentes durante el estudio que serán discutidas en el presente apartado.

\section{Características de los prejuicios, estereotipos y manifestaciones de discriminación}

En lo relacionado con los estereotipos, se identifican que los estudiantes que no

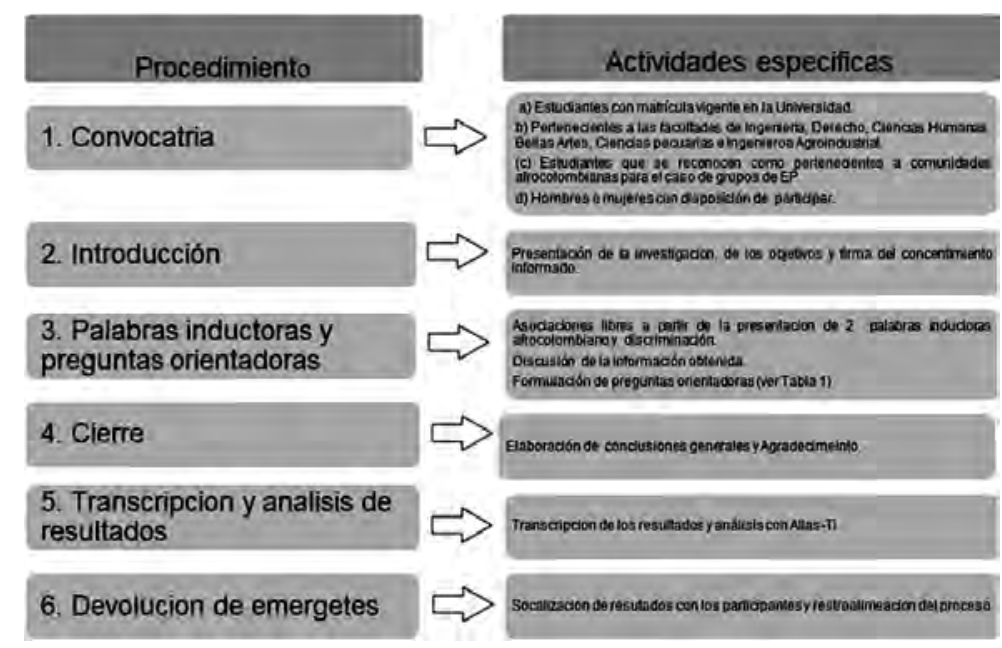

Figura 1. Protocolo para el desarrollo de grupos focales. Elaboración propia. 
Tabla 1. Preguntas orientadoras. Elaboración propia.

\section{Listado de preguntas orientadoras para grupos focales}

T: Para todos NP: Para No pertenecientes P: Para Pertenecientes

1. T. (Teniendo en cuenta los insumos de las palabras inductoras) ¿Consideran que la forma como ustedes describen a los afrocolombianos incide en la forma como son percibidos dentro del contexto universitario? ¿Por qué?

2. T. ¿Qué características se le atribuye al estudiante afrocolombiano en la Universidad de Nariño?

3. T. ¿Cómo pueden incidir las características que se atribuye al estudiante afrocolombiano en las relaciones que establece con sus compañeros dentro y fuera del aula de clases?

4. T. ¿Qué entienden por discriminación?

5. T. ¿Cuáles consideran que son las causas de la discriminación?

6. N.P ¿Teniendo en cuenta lo que ustedes consideran como discriminación, creen que en la Universidad existe algún tipo de discriminación hacia estudiantes que pertenecen a comunidades afrocolombianas? ¿Cómo se manifiesta esta discriminación?

7. N.P ¿Consideran que los estudiantes que pertenecen a comunidades afrocolombianas tienen dificultades para relacionarse con sus compañeros? ¿Por qué? P. ¿Han presentado alguna dificultad para relacionarse con sus compañeros o compañeras por el hecho de ser afrocolombiano? ¿Por qué?

8. N.P ¿Consideran que en la Universidad se generan espacios para compartir con estudiantes que pertenecen a comunidades afrocolombianas? P. ¿Consideran que en la Universidad se generan espacios para que los estudiantes que no pertenecen a su comunidad puedan compartir con los estudiantes pertenecientes a comunidades afrocolombianas?

9. T. ¿Qué propuesta realizarian para evitar que se sigan manifestando esas situaciones de discriminación en la universidad con los estudiantes pertenecientes a comunidades afrocolombianas?

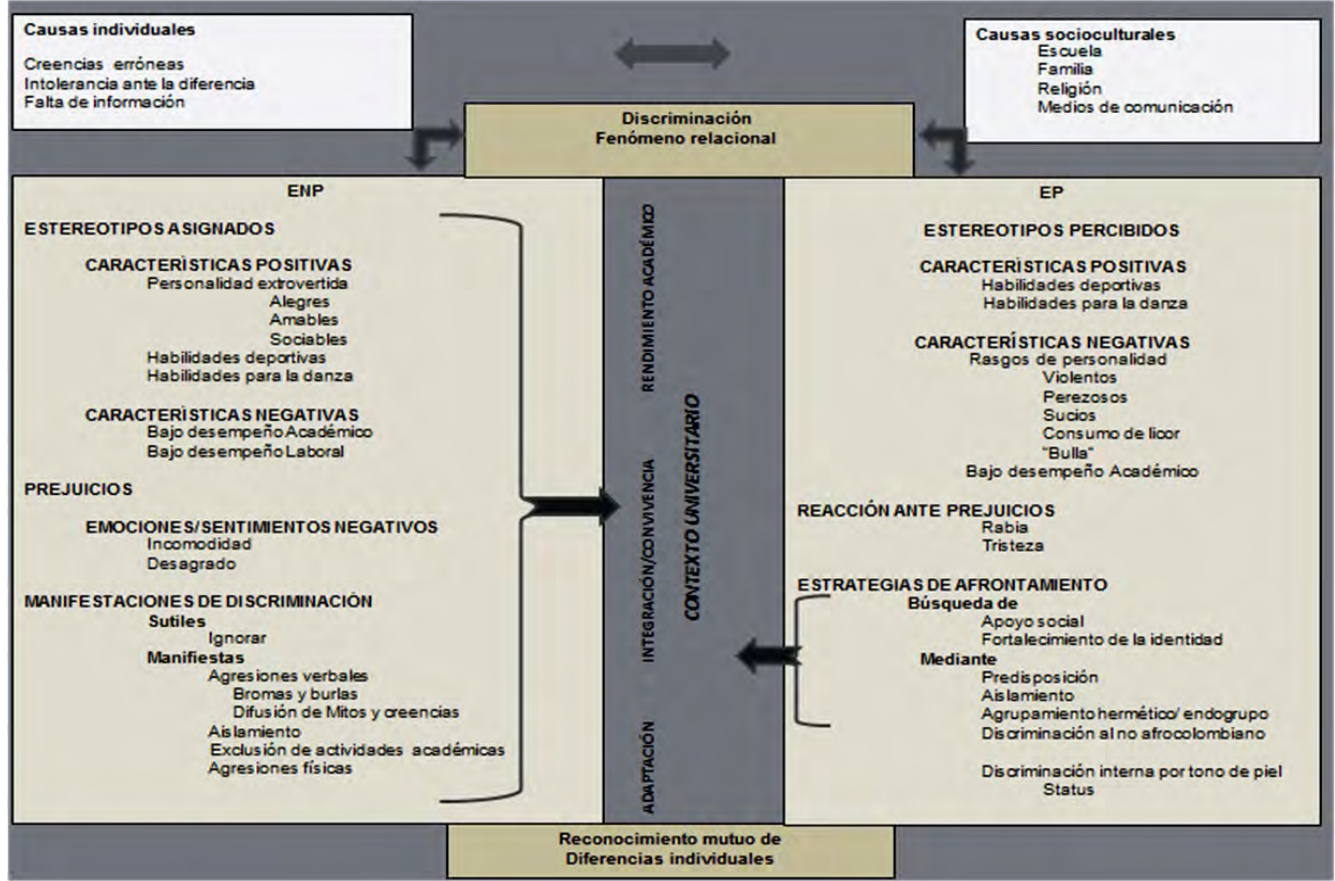

Figura 2: Categorías analíticas. Elaboración propia. 
pertenecientes a la comunidad afrocolombiana asignan características tanto positivas como negativas al estudiante afrocolombiano en el contexto universitario, las positivas se asocian con rasgos de una personalidad extrovertida destacando que son personas alegres, expresivas, efusivas, "buena gente" y sociables, así como con sus cualidades para el deporte y el baile. Al respecto, Chaparro (2007) también encontró que la alegría, la resistencia física, las habilidades para el baile y el deporte se consideran cualidades que caracterizan a aquellos sujetos que pertenecen a la "raza negra", dichas características hacen evidente un estereotipo que aunque aparentemente revela aspectos positivos del afrocolombiano, en un contexto académico implica contradicciones que no se adecuan al comportamiento esperado de un "buen" estudiante universitario, lo que se confirma al encontrar que también se les atribuyen características negativas como la falta de dedicación al trabajo y al estudio, la pereza y el facilismo las cuales se relacionan con la percepción de un bajo desempeño laboral y académico y se reflejan en frases como "Se dice que son perezosos, que les gusta dedicarse al deporte y que no quieren trabajar" ENP

Al respecto, se encontró que los estudiantes pertenecientes a las comunidades afrocolombianas reconocen y valoran positivamente que la comunidad educativa los perciba como alegres, entusiastas, destacados por sus habilidades para el baile y el deporte, sin embargo, consideran que estas características contribuyen a consolidar un estereotipo que incide negativamente en su rol como estudiantes universitarios al relacionarlos únicamente con el baile, el consumo de licor o "la buIla", en este sentido, Cunin (2003, citada por Wabgou, 2009) sostiene que a pesar de ser interpretado comúnmente como algo positivo paradójicamente el nuevo multiculturalismo

parece encerrar aún más al negro dentro de una categoría reductora e inferiorizante basada en las capaci- dades físicas asociadas de manera natural a la raza negra... incluso por sus apariencias no solo físicas sino sociales, creadas por el éxito deportivo y transmitidas por los medios (p.24)

Esto demuestra como plantea Viveros (citada en Meertens, D., Viveros M., y Arango L., 2008) que las particularidades del afrocolombiano encuentran dificultad para ser expresadas fuera de un dispositivo cultural y corporal, como si el cuerpo y la cultura fuesen los únicos capitales de los que dispusieran; de igual manera, los estudiantes pertenecientes a comunidades afrocolombianas que participaron en el estudio consideran que generalmente se percibe al afrocolombiano como violento, sucio y perezoso, características que en su conjunto develan la existencia de un estereotipo que dificulta su integración con la comunidad educativa dentro y fuera de los espacios académicos

Finalmente es necesario resaltar que los estudiantes que participaron en el estudio consideran que "no todos" los estudiantes afrocolombianos poseen las características negativas anteriormente mencionadas, por el contrario, reconocen que se destacan por su dedicación y buen rendimiento académico es decir, a pesar de que la presencia de estereotipos conduce a la "creencia de que las personas que pertenecen a otros grupos distintos al nuestro comparten en mayor medida las características que les asignamos" (Gómez, 2007, p.226) lo que desde la psicología social se conoce como homogeneidad exogrupal, en este caso es posible afirmar que el establecer vínculos afectivos y tener una convivencia prolongada con compañeros afrocolombianos en el contexto universitario facilita la ruptura de prejuicios gracias al reconocimiento de las diferencias individuales.

En relación a los prejuicios, que aquí se relacionan con el sustento afectivo de las situaciones de discriminación, se destaca la sutileza de las expresiones utilizadas 
por la mayoría de los participantes de los grupos focales al manifestar sus sentimientos negativos hacia los compañeros afrocolombianos, probablemente por temor a ser juzgados como prejuiciosos o racistas, en este punto es importante resaltar que las manifestaciones contemporáneas de discriminación hacia las comunidades afrocolombianas, no pueden ser reconocidas ni abordadas de la misma manera que hace algunas décadas, de igual manera sucede con los prejuicios que las sustentan debido a la transformación que dichas expresiones han sufrido en los últimos años y que las han hecho más sutiles y difíciles de identificar. Para Reales (2005) esto se debe a la presencia de fuertes presiones normativas y legislativas a favor de la igualdad, la tolerancia y el respeto por la diferencia que caracteriza a los estados democráticos modernos y que ha conducido a la creencia generalizada de que las actitudes prejuiciosas ante estos grupos tienden a desaparecer complejizado más su identificación.

Sin embargo junto a estas manifestaciones sutiles se identificó que algunos estudiantes no pertenecientes a las comunidades afrocolombianas reconocen sentimientos de incomodidad al compartir espacios físicos o tener encuentros directos con sus compañeros pertenecientes a comunidades afrocolombianas, sumado al desagrado que les produce convivir con ellos. Situaciones que perciben los estudiantes Afrocolombianos y que manifiestan les generar sentimientos de rabia y tristeza. Estos resultados, en concordancia con lo encontrado por Gaertner y Dovidio (citados en Angosto \& Martínez, 2004) indican que el prejuicio no se traduce necesariamente en odio y hostilidad manifiesta sino en incomodidad, inseguridad, miedo etc. observándose una tendencia a evitar el contacto intergrupal más que a manifestar conductas agresivas, lo que invita a hacer una lectura más profunda de esta problemática en los contextos universitarios tanto dentro como fuera del aula, puesto que este tipo de actitudes prejuiciosas afectan el bienestar y autoestima de quienes son sus receptores, lo cual puede incidir en el éxito académico y la integración social a este contexto.

En lo relacionado con las manifestaciones de discriminación hacia estudiantes pertenecientes a las comunidades afrocolombianas, de acuerdo a lo expresado por los dos grupos de estudiantes, en el contexto universitario se perciben conductas de discriminación que van desde las más sutiles como ignorarlos al no contestar el saludo, hasta las más directas como burlarse de su forma de vestir o hablar, cambiarse de puesto, hacer bromas o chistes pesados, difundir mitos y falsas creencias, evitar desarrollar actividades académicas con ellos e incluso infringir agresiones físicas o verbales; los estudiantes afrocolombianos reconocen que estas manifestaciones pueden afectar su autoestima al sentir que no son aceptados dentro de un contexto académico que los hace considerarse extraños y aislados, por ejemplo algunos manifiestan que suelen verse obligados a trabajar solos al momento de conformar grupos de trabajo , situación que incide en su motivación y rendimiento académico y que en el peor de los casos en interacción con otros factores puede conducir a la deserción estudiantil debido a la falta de adaptación al contexto universitario, también consideran que las situaciones de discriminación que algunos experimentan repercuten de manera negativa en los nuevos estudiantes de esta comunidad que ingresan a la universidad, quienes en algunos casos al escuchar las experiencias negativas de sus compañeros, experimentan temor al aislamiento y rechazo, incluso antes de iniciar su vida universitaria lo cual también conduce a generar en ellos prejuicios y estereotipos que los predisponen con la comunidad de acogida y pueden conducirlos a aislarse o evitar el contacto con sus compañeros reforzando así sus creencias previas, situación que si bien generalmente se trasforma positivamente mediante la interacción y convivencia da cuenta del carácter 
relacional de la problemática descrita . Al respecto Wade (1997, citado en Andrés, 2008) explica que el racismo es relacional, es decir, la diferenciación que se establece está dada por procesos relacionales entre dos o más individuos, por lo que el mismo individuo que en una interacción puede ser discriminado, también puede discriminar en una interacción con otro individuo, en este sentido, los estudiantes pertenecientes a comunidades afrocolombianas son susceptibles de recibir y generar manifestaciones de prejuicio y discriminación.

Lo anterior se constata en la presente investigación, ya que una de las características que los estudiantes no pertenecientes a comunidades afrocolombianas le atribuyen a los estudiantes afrocolombianos y que estos últimos también reconocen, es que tienden a "encerrarse" mucho con las personas de su misma comunidad y en ocasiones aíslan o "discriminan" a quien no hace parte de ella, situación que se puede constituir en una estrategia de afrontamiento ante el rechazo que perciben ya que el vínculo con un grupo de referencia proporciona seguridad y respaldo y los elementos culturales comunes pueden contribuir a la construcción de una identidad social más positiva, sin embargo, a pesar de la función social de este tipo de comportamientos también se generan situaciones de rechazo hacia la comunidad universitaria de las cuales es importante generar consciencia ya que terminan reproduciendo las mismas actitudes que se le critican, es lo que Van Dijk (2003, citado en Posso, 2008) reconoce como un momento circunstancial, es decir, que una persona discrimina o es discriminada racialmente dependiendo del momento, tiempo y contexto particular en el cual está inmerso, convirtiéndose por momentos en víctima, y en otros en agente del racismo, al respecto, se encontró por ejemplo que entre los mismos estudiantes que pertenecen a comunidades afrocolombianas se ponen de manifiesto situaciones de discriminación relacionadas con la claridad de su tono de piel siendo que aquellos con piel más oscura suelen recibir mayores manifestaciones de discriminación lo que da cuenta del color de piel como símbolo de status grupal, al respecto Murillo (1997) considera que en Colombia la mayoría de las actividades están guiadas por el color de piel; así, las personas con piel usualmente más clara son más favorecidas que las personas con color de piel más oscura. Situación que da cuenta de las raíces profundas que ha dejado en la conciencia colectiva la idea de raza y que se reproducen sin mayor crítica y reflexión incluso dentro de las mismas comunidades receptoras de situaciones de discriminación.

\section{Origen y mantenimiento de la problemática}

Entre las principales causas que atribuyen a la discriminación tanto los estudiantes pertenecientes como los no pertenecientes a comunidades afrocolombianas se encuentran: a nivel individual, la dificultad para trasformar creencias erróneas, la intolerancia ante la diferencia y la escasa o tergiversada información respecto a la cultura de esta población y a nivel socio cultural, la influencia de la educación, la familia, la religión y los medios de comunicación en el rechazo a la diversidad y la diferencia, el desconocimiento de las características particulares de la población afrocolombiana, su historia y su aporte al conocimiento y la cultura nacional y universal, causas que en su conjunto resaltan la importancia de las variables socio culturales al ser estos fuertes dispositivos para la creación, difusión y mantenimiento de los estereotipos y prejuicios que promueven situaciones de discriminación, aspectos que pueden identificarse en el lenguaje cotidiano de nuestra sociedad, al respecto Becerra y Gonzales (2013) analizando el papel del lenguaje en relación al concepto de raza consideran que

...el lenguaje... la palabra hablada, se convierte en el medio que permite establecer criterios de exclusión, fragmentación de identidades, burlas, miedo, en general una 
posición de inferioridad frente a los Otros (p. 271-272)

En este sentido, cabe considerar los planteamientos de Brion Davis (1970, citado en Wabgou, 2009) quien resalta cuatro causas para que durante la colonia los negros y no los indígenas fueran esclavizados: en primer lugar el hecho de que los africanos fueran vistos como paganos desde el sistema de conocimiento europeo justificando la esclavización como mejor manera de convertirlos al cristianismo, la segunda las interpretaciones bíblicas que argumentaban la esclavitud de los negros debido a una maldición de Noé, la tercera la vinculación que sostenía la cultura europea del color de piel de los africanos con el mal, el demonio y la sociedad, y la cuarta que la esclavización africana en Europa ya existía, de manera que trasladarla a América era simplemente dar continuidad a una práctica ya legitimada. Los elementos que señala este autor, ayudan a comprender cómo el discurso trasmitido por la religión ha contribuido al mantenimiento de prejuicios hacia la población afrocolombiana que aún prevalecen en el imaginario colectivo, pues ello ha permitido la permanencia del poder en beneficio de ciertos intereses.

Asimismo, la escuela ha contribuido a este fenómeno mediante la difusión de una imagen estereotipada del afrocolombiano que se transmite en los libros de texto y el desconocimiento de su aporte al conocimiento y la cultura nacional, lo cual es confirmado por el Ministerio de Educación Nacional (s.f) al referir que el análisis de textos escolares devela una valoración discriminatoria de géneros, clases, razas y etnias, lo que demuestra la necesidad de hacer propuestas alternativas en materiales de consulta y de apoyo didáctico que reconozcan la diversidad étnica y cultural de los estudiantes.

Respecto a los medios de comunicación, el Observatorio Contra la Discriminación y el Racismo (2012), muestra que estos registran solo entre 2 y 9 por ciento las noticias, hechos o acontecimientos que involucren a la población afrocolombiana, además da a conocer que con frecuencia promueven una caracterización determinada de la raza "negra", ya sea como manifiesto de una discriminación estructural o como estrategias intencionadas de exclusión racial. En este sentido lo "negro" y lo "afro" sólo es incluido en cuanto a la construcción de valores y estereotipos que comúnmente han sido asociados con fuerza física y talento musical. Asimismo la imagen del afrocolombiano se muestra asociada a la miseria, el crimen, la prostitución, el deporte y la música.

Alternativas para el abordaje de la problemática

Entre las estrategias que sugieren el conjunto de estudiantes para el abordaje de estas problemáticas se destaca la generación de espacios y políticas institucionales dirigidas a promover y divulgar el aporte de las comunidades afrocolombianas al conocimiento universal y la cultura nacional, así como la reflexión de los procesos históricos, sociales y políticos que han sustentado los prejuicios y estereotipos hacia esta población.

Para entender la discriminación se requiere reconocer cómo históricamente se ha respondido a la pregunta sobre el otro diferente, dado que la historia de un país, una región o una familia influyen en el comportamiento de los individuos. Los recuerdos e ideas del otro de épocas anteriores permanecen conscientes o latentes en nosotros... están guardados y ordenados en nuestra mente, pero la memoria colectiva borra determinadas imágenes y conserva otras, las cuales magnifica o deforma; por lo tanto, se hace necesario reconocer estas elecciones, aunque se califiquen como arbitrarias o injustas, pues permite entender nuestro presente. (García, 2002, p.7).

Cabe resaltar que los estudiantes no pertenecientes a las comunidades afrocolombianas, aunque manifiestan reconocer la importancia de respetar la diferencia y 
generar procesos a favor de los denominados grupos minoritarios se muestran en desacuerdo con los beneficios y cupos especiales que otorga la universidad a estudiantes de comunidades indígenas y afrocolombianas como parte de las acciones afirmativas o de discriminación positiva que realiza el estado y sus instituciones para garantizar el acceso equitativo a la educación superior, por lo tanto es importante que los estudiantes no pertenecientes a estas comunidades conozcan las razones de este tipo de políticas, de manera que no las perciban como discriminación generando rechazo hacia dichas comunidades, en este punto se debe considerar que son los diferentes actores de la comunidad académica docentes, estudiantes y administrativos los llamados a generar estos espacios de reflexión y convivencia que contribuyan a eliminar las concepciones erróneas que se tienen frente a la población afrocolombiana, en el marco de una política universitaria que garantice el cumplimiento y seguimiento de estos propósitos.

\section{Reflexiones finales}

El estudio permite concluir que la Universidad de Nariño tiene una deuda pendiente con la comunidad afrocolombiana que va desde la falta de reconocimiento de sus aportes a la historia, la cultura y el conocimiento hasta la indiferencia frente a prácticas cotidianas que ocurren en el contexto universitario donde consciente o inconscientemente se reproducen prejuicios y estereotipos hacia esta población, los cuales generan manifestaciones de exclusión o discriminación que afectan su calidad de vida, dignidad y bienestar, manifestaciones que pueden tomar forma de agresiones físicas o verbales pero que con mayor frecuencia adquieren formas de rechazo sutil que hacen más difícil la identificación del problema, contribuyendo a su silenciamiento e invisibilización.

El desconocimiento de las circunstancias históricas, políticas y socioeconómi- cas de la población afrodescendiente y su silenciamiento en el discurso educativo contribuye a invisibilidad los nuevos lenguajes que adquiere la discriminación étnico racial, lenguajes que se hacen cada vez más difíciles de reconocer al camuflarse entre un discurso de aparente aceptación y respeto por la diferencia, pues incluso tras el reconocimiento aparentemente positivo de las habilidades físicas, deportivas y artísticas del afrodescendiente, se oculta el desconocimiento de su aporte a otras áreas del conocimiento situación que en este caso particular, afecta la percepción social sobre el desempeño laboral y académico de los estudiantes universitarios afrocolombianos lo cual puede generar barreras actitudinales no solo dentro sino fuera de la comunidad universitaria, que más adelante representen obstáculos y desventajas a la hora de acceder a derechos fundamentales como la educación y el trabajo.

Se evidencia que independientemente del nivel educativo, la categoría de raza permanece fuertemente anclada a la formación de nuestra subjetividad, esta categoría se instaura en un discurso de ordenamiento social por el color de la piel que incluso comparten y legitiman algunos estudiantes afrocolombianos al reconocer que se presentan casos de discriminación entre ellos mismos relacionados con el tono de su piel, esto indica que al ser el prejuicio, el estereotipo y la discriminación fenómenos psicosociales que se construyen en relación con el Otro, ser receptor de estas situaciones por el color de piel, no exime a los sujetos de reproducirlas en otros contextos y circunstancias, por lo que también se hace necesario reflexionar la problemática desde las actitudes de las propias comunidades afrocolombianas en las que no solo se reconozca su posición como receptores de la misma sino su poder trasformador como agentes de cambio social.

La dimensión relacional de la problemática descrita se hace evidente incluso en las formas de afróntala pues en el caso 
de algunos estudiantes pertenecientes a la comunidad afrocolombiana la predisposición, el aislamiento y el hermetismo endogrupal generado por las barreras establecidas al agruparse únicamente con compañeros pertenecientes a la comunidad afrocolombiana, más que erradicar la problemática, termina reproduciendo los mismos fenómenos al generar situaciones que hacen que los estudiantes no pertenecientes a su comunidad se sientan también excluidos, discriminados y estereotipados lo cual impide la trasformación de estas situaciones pues al limitar las posibilidades de interacción se obstaculiza el reconocimiento de las diferencias individuales que permite la trasformación de prejuicios y estereotipos; esto indica que es solo poniéndose en relación directa con la alteridad y permitiendo esta relación cómo se puede construir una legitima aceptación de la diversidad.

Como recomendación general se considera que el respaldo institucional y el establecimiento de sistemas de control y denuncia en el contexto Universitario se pueden constituir en mecanismos efectivos para visibilizar problemáticas relacionadas con prejuicios, estereotipos y situaciones de discriminación, para ello es importante diseñar políticas que en el caso particular de la universidad de Nariño deben ir más allá de la asignación de beneficios y cupos especiales a los estudiantes pertenecientes a minorías étnicas e incluir la reflexión profunda de las dinámicas que adquiere la diversidad cultural dentro y fuera del aula de clase, es decir en la convivencia cotidiana, a fin de generar las estrategias pertinentes para su abordaje y su respectiva prevención y seguimiento, ya que el rol de la universidad no solo se debe limitar a la transmisión de conocimientos sino que implica un compromiso con la transformación social y la búsqueda de bienestar y equidad para las diferentes poblaciones que conforman el tejido social colombiano.

\section{Bibliografía}

\section{Fuentes}

Andrés, Lydia. (2008). Racismo en comunidades de élite: un estudio de caso. Tesis de maestría en ciencias sociales con mención en antropología no publicada. Facultad Latinoamericana de Ciencias Sociales - sede Ecuador.

Asamblea Nacional Constituyente. (1991). Constitución política de Colombia de 1991.

Angosto, Josefa María y Martinez Carmen. (2004). Dimensiones y determinantes de la intención de contacto hacia exogrupos. Revista latinoamericana de psicología. Interamerican journal of psychology, ISSN 00349690, Vol. 38, №. 2, 2004 , págs. 181-190.

Becerra Gonzáles, Nini Jhohanna y Gonzáles Gonzáles, Miguel Alberto. (2013). Lenguajes del poder: los afrodescendientes vistos a través de las canciones de salsa. Revista Plumilla Educativa Nro 12. Manizales: Universidad de Manizales.

Cavelier, Catalina. (2007). El racismo desde la academia, contexto académico y aproxi- maciones a la problemática del racismo y la discriminación racial en el ámbito de las Ciencias Sociales en Colombia. En: http:// www.iidh.ed.cr/BibliotecaWeb/Varios/Documentos/BD 945261041/Racismoacademia_Colombia.pdf?url=/BibliotecaWeb/ Varios/Documentos/BD_945261041/ Racismoacademia_Colombia.pdf (Recuperado en septiembre 05 de 2013).

Castillo Guzman, Elizabeth y Caicedo Ortiz, Jose Antonio. (2014) Entre invisibilidad y reconocimiento. Luchas afrocolombianas por la educación en el siglo XX. En: file:///C:/ Users/pc/Desktop/ARTICULO\%20AFROCOLOMBIANOS/Educaci\%C3\%B3n+Mov iminto+Afrocolombiano.pdf. (Recuperado en Enero 22 de 2015)

Chaparro, Julie Andrea. (2007). "Es que tenía que ser negro": estereotipos y relaciones sociales. Latin american network information center. En: http://lanic.utexas.edu/project/etext/lilas/ilassa/2009/chaparro.pdf. (Recuperado en Diciembre 2 de 2012). 
Congreso de la República de Colombia (2011). Ley 1482 de 2011: por la cual se modifica el Código Penal y se establecen otras disposiciones. En: http:// www.observatorioweb.org/ver_leyes. php?codigo $=140 \&$ seccion $=15$. (Recuperado en Noviembre 2 de 2014)

Congreso de la Republica de Colombia (1993). Ley 70 de 1993: Por la cual se desarrolla el artículo transitorio 55 de la Constitución Política. En: http://www.minagricultura.gov. co/archivos/ley_70_de_1993.pdf (Recuperado en Octubre 6 de 2014 )

García O’meany, Margarita. (2002). Yo no soy racista, pero....: Justificando la discriminación. Barcelona: Intermón Oxfam.

Gómez Jiménes, Angel. (2007). Estereotipos. En Cejudo, José Manuel (Ed.). Psicología social. Madrid, España: McGRAW-HILL

Gross, Richard D. (2004). Psicología: La ciencia de la mente y la conducta. México; Manual Moderno.

Hernández, Gloria. y Meza, Adriana. (2008). Manifestaciones de discriminación etnoracial hacia estudiantes afrocolombianos de la Universidad de Nariño. Pasto: Manuscrito no publicado. Universidad de Nariño.

Meertens, Donny, Viveros Mara, y Arango Luz Gabriela. (2008). Discriminación étnico-racial, desplazamiento y género en los procesos identitarios de la población "negra" en sectores populares de Bogotá. En: Zabala, María del Carmen. Pobreza, exclusión social y discriminación étnicoracial en América Latina y el Caribe. Bogotá: Colombia.

Meneses Copete, Yeison Arcadio. (2013). Representaciones sociales sobre etnoeducación y cátedra de estudios afrocolombianos en la formación del profesorado. En: file:///C:/Users/pc/Desktop/ARTICULO\%20 AFROCOLOMBIANOS/representaciones\%20profesorado.pdf. (Recuperado en Enero 20 de 2015).

Ministerio de Educación nacional (1998). Decreto 1122: por el cual se expiden normas para el desarrollo de la Cátedra de Estudios Afrocolombianos, en todos los establecimientos de educación formal del país y se dictan otras disposiciones. En: http://www.mineducacion.gov.co/1621/articles-86201_archivo_pdf.pdf (Recuperado en Octubre $\overline{15}$ de 2012).
Ministerio de Educación Nacional. (s.f). Cátedra Estudios Afrocolombianos. En: http://www.mineducacion.gov.co/1621/ articles-89869_archivo_pdf1.pdf. (Recuperado en Octübre 19 de 2012).

Molero Alonso, Fernando Jorge. (2007). El estudio del prejuicio en la psicología social: definición y causas. En: Cejudo, José Manuel. Psicología social. Madrid, España: McGraw-Hill

Murillo Murgueitio, Antonio José. (1997). Soy afro-colombiano. ( $1^{\text {a }}$ edición). Bogotá; Editorial Linotipia Bolívar.

Organización de las Naciones Unidas. (2012). Unidos contra el racismo, la discriminación racial, la xenofobia y las formas de y las formas conexas de intolerancia. En: http:// www.un.org/es/letsfightracism/pdfs/united against_racism_for_web.pdf (Recuperado en Enero 23 de 2015)

Observatorio de Discriminación Racial, (2012). Segundo informe: Discriminación laboral en Cali En: http://www.mininterior.gov.co/ sites/default/files/segundo_informe_observatorio_30_septiembre_de_20121.pdf (Recuperado en Enero 25 de 2015)

Oskamp, Stuart. (1991). Racisme and prejudice. En: Oskamp, Stuart (Ed.). Attitudes and opinions. Englewood Cliffs; NJ: Prentice Hall.

París Pombo, María Dolores. (2002). Estudios sobre el racismo en América Latina. En: http://dialnet.unirioja.es/servlet/ articulo?codigo $=4151818$ (Recuperado en Octubre15 de 2014)

Posso, Jeanny. (2008). Mecanismos de discriminación étnico-racial, clases sociales y género: La inserción laboral de mujeres negras en el servicio doméstico de Cali. En Argüelles, María del Carmen Zabala. Pobreza, exclusión social y discriminación étnico-racial en América Latina y el Caribe. Bogotá: Siglo del Hombre Editores.

Prieto Rodriguez, M. Ángeles y March Cerda, Joan Carles. (2002). Paso a paso en el diseño de un estudio mediante grupos focales. En: http://www.sciencedirect.com/ science/article/pii/S0212656702705854 (Recuperado en Julio 15 de 2013)

Reales Jiménez, Leonardo. (2005). Racismo y políticas públicas en Colombia. El caso afrocolombiano En: http://www.eipcifedhop. org/EIPColombia/2005/afro_colombiens/ 
Racismo.pdf (Recuperado el 22 de Octubre de 2009)

Restrepo, Eduardo. (2003). Entre arácnidas deidades y leones africanos: Contribución al debate de un enfoque afroamericanista en Colombia. Tabula Rasa Nro.1. Bogotá: Universidad Colegio Mayor de Cundinamarca.

Restrepo, Eduardo. (2004). Hacia los estudios de las Colombias negras. En: Pardo Rojas, Mauricio; Mosquera, Claudia y Ramírez, María Clemencia (Eds). Panorámica afrocolombiana. Estudios sociales en el Pacífico. Bogotá: ICANH Universidad Nacional de Colombia.

Reyes, Tomás. (1999). Métodos cualitativos de investigación: Los grupos focales y el estudio de caso. Forum Empresarial Vol 4. Puerto Rico: Centro de Investigaciones Comerciales e Iniciativas Académicas de la Facultad de Administración de Empresas de la Universidad de Puerto Rico.

Rodríguez Zepeda, Jesús. (2005). Definición y concepto de la no discriminación. El Cotidiano Vol 21.Mexico: Universidad Autónoma Metropolitana. Azcapotzalco.

Viáfara López, Carlos Augusto y Urrea Giraldo, Fernando (2006). Efectos de la raza y el género en el logro educativo y estatus socio-ocupacional para tres ciudades colombianas. En: http://economia. uniandes.edu.co/investigaciones_y_publicaciones/CEDE/Publicaciones/Revista_Desarrollo_y_Sociedad/Ediciones/ revista_desarrollo_y_sociedad_no_58/ efectos_de_la_raza_y_el_genero_en_el_ logro_educativo_y_estatus_socio_ocupacional_para_tres_ciudades_colombianas (Recuperado el 15 de Septiembre de 2014)

Viveros, Mara. (2007). Discriminación racial, intervención social y subjetividad. Reflexiones a partir de un estudio de caso en Bogotá. Revista de Estudios Sociales Nro 27. Bogota: Universidad de los Andes.

Wabgou, Maguemati. (2009). Voces de la población afrocolombiana en la localidad de Kennedy. Bogotá: Universidad Nacional de Colombia.

\section{Referencias}

Agudelo, Carlos Efrén. (2004). No todos vienen del río: construcción de las identidades negras urbanas y movilización política en
Colombia. En: Restrepo, Eduardo y Rojas, Axel. Conflicto e (in)visibilidad. Retos en los estudios de la gente negra en Colombia. Popayán, Colombia: Universidad del Cauca.

Alonso, María José, Navarro, Raquel y Vicente, Lidón. (s.f). Actitudes hacia la diversidad entre estudiantes universitarios. España: Universitat Jaune.

Asamblea general de las naciones Unidas. (1965). Convención Internacional sobre la Eliminación de todas las Formas de Discriminación Racial disponible en http:// www.sre.gob.mx/images/stories/dgpme/ acuerdos/coninter_eliminar_formasdiscriminacion.pdf

Bedmar Moreno, Matías. (2002). La educación social contra la exclusión: interculturalidad. Estudios sobre las culturas contemporáneas Vol VIII. Mexico: Universidad de Colima.

Careaga Pérez, Gloria. (2003). El racismo y el sexismo en las expresiones sexuales. Revista de estudios de Género la ventana. En: http://redalyc.uaemex.mx/redalyc/ src/inicio/ArtPdfRed.jsp?iCve $=88401812$ (Recuperado en Noviembre 25 de 2012)

Lacerda Teixeira, Ana María. (2010). El prejuicio racial en Brasil: medidas comparativas. En: http://www.scielo.br/scielo. php?pid=S0102$71822010000100005 \&$ script $=$ sci_arttext (Recuperado en Octubre 6 de 2014)

Lacerda Teixeira, Ana María y Molero Alonso, Fernando. (2008). El prejuicio racial en Brasil: nuevas medidas y perspectivas. En: http://www.redalyc.org/articulo. oa? $i d=309326698013$. (Recuperado en Octubre 6 de 2014)

Martinez Hoyos, Maria Fernanda y Rodríguez Pabón, Diana Milena. (2012). Universidad y diversidad cultural. Diálogos imperfectos. Revista Plumilla Educativa Nro 10. Manizales: Universidad de Manizales.

Ministerio de Educación nacional (2004). Directiva Ministerial 011 de 2004: Orienta la prestación del servicio educativo en entidades territoriales que atienden población afrocolombiana y raizal. En: http://www.colombiaaprende.edu.co/html/ mediateca/1607/articles-88152_archivo. pdf (Recuperado en Octubre 15 de 2012).

Molero Alonso, Fernando Jorge. (2007 b). Prejuicio y estigma: efectos y posibles so- 
luciones. En: Cejudo, José Manuel. Psicología social. Madrid, España: McGraw-Hill

Mosquera Rosero-Labbé, Claudia y León Díaz, Ruby Esther. (2009) Acciones Afirmativas y ciudadanía diferenciada étnicoracial negra, afrocolombiana, palenquera y raizal. Entre Bicentenarios de las Independencias y Constitución de 1991. Bogotá: Universidad Nacional de Colombia.

Ojeda, Elizabeth; González, Patricia; Martínez, María Fernanda; Rodríguez, Diana; Caicedo, Ana; Pantoja, Mauricio y Zambrano; Christian. (2012). Prejuicios y estereotipos entre estudiantes universitarios Pertenecientes y No Pertenecientes a Comunidades Indígenas, Afrocolombianas y Homosexuales de la Universidad de $\mathrm{Na}$ riño. Informe de Investigación presentada en el Concurso de Investigación Docente Alberto Quijano Guerrero. Pasto. Universidad de Nariño.

Rodríguez Garavito, Cesar; Alfonso Sierra, Tatiana y Cavelier Adarve, Isabel. (2009). Raza y derechos humanos en Colombia Informe sobre discriminación racial y derechos de la población afrocolombiana. Bogotá: Ediciones Uniandes.

Serna, Sonia. (2011).Vivir de los imaginarios del mar: restaurantes y estereotipos sobre el Pacífico en Bogotá.Tabula Rasa Nro. 14. Bogotá: Universidad Colegio Mayor de Cundinamarca.

Viáfara, Carlos. (2010). Movimiento Nacional por los Derechos Humanos de las Comunidades Afrocolombianas CIMARRON. Colombia: Informe Anual: Situación de Derechos Humanos de la Población Afrocolombiana 2009-2010. Bogotá: Agencia
Española de Cooperación Internacional para el Desarrollo, Unión Europea y Acción Social.

Viáfara López, Carlos Augusto; Urrea Giraldo, Fernando y Correa Fonnegra, Juan Byron. (2005).Desigualdades sociodemográficas y socioeconómicas, mercado laboral y discriminación étnico-racial en Colombia: análisis estadístico como sustento de Acciones Afirmativas a favor de la población afrocolombiana. En Mosquera, Claudia y León, Ruby (Eds). Acciones Afirmativas y ciudadanía diferenciada étnicoracial negra, afrocolombiana, palenquera y raizal. Bogotá: Universidad Nacional de Colombia.

Villa, Wilmer y Grueso, Arturo (2008). Emergencias posibles desde la construcción de la interculturalidad. Diversidad, Interculturalidad y construcción de ciudad. Bogota: D'vinni S.A.

Wabgou, Maguemati; Arocha Rodriguez, Jaime; Salgado Cassiani, Aiden Jose y Carabalí Ospina, Juan Alberto. (2012). Movimiento Afrocolombiano, Negro, Raizal y Palenquero: El largo camino hacia la construcción de espacios comunes y alianzas estratégicas para la incidencia política en Colombia. Bogotá: Universidad Nacional de Colombia.

Walsh, Catherine. (2008). Interculturalidad crítica Pedagogía de-colonial. Diversidad, Interculturalidad y Construcción de Ciudad. Bogotá: D'vinni S.A.

Worchel, Stephen; Cooper, Joel; Goethals, George y Olson, James. (2002). Prejuicios y estereotipos, Psicología social. Mexico: Thompson Learning. 
Plumilla Educatival

104 • Instituto Pedagógico 